\title{
A Clinicopathological Study on Cardiac Lesions in 64 Cases of Disseminated Intravascular Coagulation
}

\author{
Masaya Sugiura, M.D., Keisuke Hiraoka, M.D., \\ Shin-ichiro Ohkawa, M.D., Keiji Ueda, M.D., \\ Tamotsu Matsuda, M.D., * and \\ Mototaka Murakami, M.D.
}

SUmmary

Diagnosis of disseminated intravascular coagulation (DIC) was made in 64 cases $(16.2 \%$ ) among a total of 395 autopsy cases. There were 31 men and 33 women. Their ages ranged from 31 to 91 years (mean 76.3). Underlying diseases were mainly malignancy and sepsis.

Fresh cardiac lesions were found in 40 cases $(62.5 \%)$. Coronary thrombosis was found in 13 cases $(20.3 \%)$ and myocardial necrosis in 24 cases $(37.5 \%)$, with acute myocardial infarction in 9 and focal necrosis in 15 . Nonbacterial thrombotic endocarditis was found in 17 cases $(26.6 \%)$, mural thrombi in $11(17.2 \%)$, and bleeding of the heart in $11(17.2 \%)$. Platelet count, fibrinogen and euglobulin lysis time were not correlated with myocardial necrosis nor coronary thrombosis. Increase of fibrin degradation products correlated with the presence of coronary thrombosis with or without myocardial necrosis.

DIC was found with a high incidence in the aged, and many of them were complicated with fresh cardiac lesions. Development of acute myocardial infarction depends on the small thrombi in the severe stenosis of the main coronary arteries or on the multiple microthrombi in the peripheral coronary branches.

\section{Additional Indexing Words :}

Coronary thrombosis Myocardial infarction Nonbacterial thrombotic endocarditis

$\mathrm{D}$ ISSEMINATED intravascular coagulation (DIC) was noted to be often complicated with malignancy and sepsis ${ }^{1-4)}$ and its high incidence has been stressed in the aged. 4) Pathological findings in DIC were thrombus formation, ischemic necrosis and bleeding, which were frequently found in the

From the Department of Internal Medicine, Tokyo Metropolitan Geriatric Hospital (Yoiku-in), Itabashi, Tokyo 173, Japan.

* Department of Clinical Physiology, Tokyo Metropolitan Institute of Gerontology, Itabashi, Tokyo.

Received for publication April 27, 1976. 
skin, kidney, brain, and lung. ${ }^{1,31,5)}$ Few cardiac lesions, however, have been reported. ${ }^{1,51,6)}$ and we have not known any reports of myocardial infarction with DIC. The purpose of this study was clinicopathological examination of the cardiac lesions found in DIC.

\section{Materials and Methods}

A total of 64 cases (16.2\%) of DIC was diagnosed among 395 cases of consecutive autopsy from July, 1972 to June, 1974 in Yoiku-in Hospital. Their ages ranged from 31 to 91 years with an average of 76.3 years, and all but 2 were over 60 years. There were 31 men and 33 women. Diagnostic criterion of DIC was a presence of consumption coagulopathy, evaluated by a decrease of platelet count (less than $10^{5} / \mathrm{mm}^{3}$ ) and fibrinogen (less than $200 \mathrm{mg} / 100 \mathrm{ml}$ ), prolongation of euglobulin lysis time (ELT) and increase of the fibrin degradation products (FDP) (more than $20 \mu \mathrm{g} / \mathrm{ml}$ ).

Pathological examinations of the heart included dissection of the coronary arteries with $5 \mathrm{~mm}$ interval towards the peripheral branches, and the patency of the lumen was recorded using the count described previously; ${ }^{7}$ that is count 5 in cases of $100 \%$ obstruction, 4 in $75 \%, 3$ in $50 \%, 2$ in $25 \%$, and 1 in slight stenosis. Stenotic index was a sum of the highest count of the 3 vessels. Gross and histological observations were made concerning the presence of thrombi and fresh myocardial necrosis. Size of myocardial infarction was classified into 3 groups $;^{71}$ large (longer diameter of more than $5 \mathrm{~cm}$ ), middle-sized $(4.9 \mathrm{~cm}$ to $2.1 \mathrm{~cm}$ ) and small (less than $2 \mathrm{~cm}$ ) infarction. Small necrosis (longer diameter of less than $1.0 \mathrm{~cm}$ ) was not included in myocardial infarction. Observations were also made on the presence of nonbacterial thrombotic endocarditis, mural thrombus, and hemorrhage in the heart.

\section{Results}

\section{Incidence of DIC}

DIC was found in 64 cases among a total of 395 with an incidence of $16.2 \%$. Underlying diseases were malignancy in 28 cases, sepsis 8, combination of malignancy and sepsis 9, pneumonia 6, and others 13 .

II. Incidence of fresh cardiac lesions (Table I)

Fresh cardiac lesions were found in 40 cases $(62.5 \%)$. Coronary thrombosis was found in 13 cases $(20.3 \%$ ), which was always complicated with fresh necrosis. Nonbacterial thrombotic endocarditis was found in 17 cases $(26.6 \%)$, which was located at the aortic valve in 10 cases and at the mitral valve in 10. Eight cases of them also had coronary thrombosis. Mural thrombi were noted in 11 cases $(17.2 \%)$, which were located in the left ventricle in 10 , right atrium in 2, and right ventricle in 1 . Four cases out of them 
had coronary thrombosis. Myocardial necrosis was found in 24 cases $(37.5 \%) ; 9$ of them showed fresh myocardial infarction and 15 small focal necrosis. Cardiac bleeding was noticed in 11 cases $(17.2 \%)$; 9 of them had petechiae and/or small bleeding in the pericardium and/or endocardium. Massive intramyocardial bleeding was found in only 2 cases A total of 24 cases did not show any fresh cardiac lesions.

Table I. Fresh Cardiac Lesions in D.I.C.

\begin{tabular}{|c|c|c|}
\hline Total & 40 & $(62.5 \%)$ \\
\hline Coronary Thrombosis & 13 & $(20.3 \%)$ \\
\hline Nonbacterial Thrombotic Endocarditis & 17 & $(26.6 \%)$ \\
\hline Aortic & 10 & $(15.6 \%)$ \\
\hline Mitral & 10 & $(15.6 \%)$ \\
\hline Mural Thrombus & 11 & $(17.2 \%)$ \\
\hline Left Ventricle & 10 & $(15.6 \%)$ \\
\hline Right Ventricle & 1 & $(1.6 \%)$ \\
\hline Right Atrium & 2 & $(3.1 \%)$ \\
\hline Myocardial Necrosis & 24 & $(37.5 \%)$ \\
\hline Myocardial Infarction & 9 & $(14.1 \%)$ \\
\hline Small Necrosis & 15 & $(23.4 \%)$ \\
\hline Bleeding & II & $(17.2 \%)$ \\
\hline
\end{tabular}

Table II. Pathologic Findings in DIC with Coronary Thrombosis and Myocardial Necrosis

\begin{tabular}{|c|c|c|c|c|c|c|c|c|}
\hline \multirow[b]{2}{*}{ No. } & \multirow[b]{2}{*}{ Cardiac Lesions } & \multirow[b]{2}{*}{ SI } & \multicolumn{3}{|c|}{ Site \& Size of Thrombosis } & \multirow[b]{2}{*}{ Verruca } & \multirow{2}{*}{$\begin{array}{c}\text { Mural } \\
\text { Thrombus }\end{array}$} & \multirow{2}{*}{ Bleeding } \\
\hline & & & $\begin{array}{l}\text { Main } \\
(\mathrm{mm})\end{array}$ & $\begin{array}{c}\text { Branch } \\
(\mathrm{mm})\end{array}$ & $\begin{array}{c}\text { Arteriole } \\
(/ 1)\end{array}$ & & & \\
\hline 1 & MI large (A-S) & 13 & 0.8 & & & - & $+\mathrm{LV}$ & - \\
\hline 2 & MI large (L) & 4 & & 1.2 & 350 & $+a$ & - & - \\
\hline 3 & MI large (A-S-I) & 4 & & 1.0 & 100 & - & $+\mathrm{LV}$ & - \\
\hline 4 & MI middle (L) & 13 & 0.9 & & $80-380$ & - & $-\ldots$ & - \\
\hline 5 & MI middle (I) & 13 & & 1.3 & & $+\mathrm{m}$ & - & - \\
\hline 6 & MI middle (I) & 5 & & 2.1 & $80-130$ & $+\mathrm{m}$ & $+\mathrm{LV}$ & - \\
\hline 7 & MI small (I) & 8 & & 2.0 & $70-110$ & $+\mathrm{m}$ & - & - \\
\hline 8 & Small Necrosis (d) & 13 & & 1.5 & 200 & $+\mathrm{m}$ & - & - \\
\hline 9 & Small Necrosis (I) & 12 & & 1.5 & $100-400$ & $+\mathrm{m}$ & - & - \\
\hline 10 & Small Necrosis (d) & 11 & & & $50-200$ & - & $+\mathrm{LV}, \mathrm{RA}$ & - \\
\hline 11 & Small Necrosis (d) & 11 & & & $40-200$ & $+a$ & - & - \\
\hline 12 & Small Necrosis (d) & 8 & & & $70-120$ & - & - & $+\mathrm{LV}$ \\
\hline 13 & Small Necrosis (I-L) & 6 & & & 100 & $+m$ & - & - \\
\hline
\end{tabular}

Abbreviations: SI; Stenotic Index, MI; Myocardial Infarction, A; anterior, S; septal, L; lateral, I; inferior, d; diffuse, $\mathrm{m}$; mitral valve, a ; aortic valve, LV; Left Ventricle, RA; Right Atrium. 
III. Relationship between coronary thrombosis and myocardial necrosis (Table II)

All of 13 cases of coronary thrombosis had some types of myocardial necrosis, and other 11 cases showed also myocardial necrosis without coronary thrombosis. Myocardial lesions found in 13 cases of coronary thrombosis were large myocardial infarction in 3, middle-sized infarction in 3, small infarction in 1, and small focal necrosis in 6 . Coronary thrombosis in the main arteries (diameter of thrombi of 0.8 and $0.9 \mathrm{~mm}$ ) resulted in a large and a middle-sized myocardial infarction in 2 cases (Cases 1 and 4). Thrombosis in the coronary branches (diameter of 1.0 to $2.1 \mathrm{~mm}$ ) in 7 cases resulted in large infarction in 2, middle-sized infarction in 2, small infarction in 1, and focal necrosis in 2. Multiple microthrombi in the intramyocardial small arteries and arterioles were from 40 to $400 \mu$ in diameter, and found in 11 cases. Among them microthrombi alone could result in small necrotic foci in 4 cases.

Pathogenesis of large myocardial infarction in 3 cases was analyzed. In 1 case a thrombus was found in the severely stenotic segment of the left anterior descending artery, resulting in a massive large anterior infarction (Case 1). In the other 2 cases there was no marked coronary stenosis. In 1 of them thrombosis was found in the peripheral branches of the left circumflex

Table III. Clinical Findings in DIC

\begin{tabular}{|c|c|c|c|c|c|c|}
\hline No. & Case & Age & Sex & Underlying Diseases & EGG Changes & Remark \\
\hline 1 & T. S. & 74 & $\mathrm{~F}$ & Pncumonia, Uremia & $\mathrm{QS}_{\mathrm{V} 4}, \mathrm{R}_{\mathrm{V} 2,3} \downarrow$ & H \\
\hline 2 & K. T. & 85 & M & Cancer of Stomach & $Q_{\mathrm{V} 1-4}$ & + \\
\hline 3 & K. N. & 70 & $\mathbf{M}$ & D.M., Hypertension & LBBB, $\mathrm{ST}_{\mathrm{V} 1-4} \uparrow$ & $H$ \\
\hline 4 & Y.S. & 76 & $\mathrm{~F}$ & Cancer of Stomach, Sepsis & $\begin{array}{l}\text { af Tachyc., Trans. } \mathrm{QS}_{\mathrm{V}}, \mathrm{R}_{\mathrm{V} 1-3 \downarrow}, \\
\mathrm{ST}_{\mathrm{V}, 5,5} \uparrow \text {, Trans. VT. }\end{array}$ & + \\
\hline 5 & K.S. & 76 & $\mathrm{~F}$ & Cancer of Gall Bladder & $\begin{array}{l}Q_{I I, a V F}, \text { af Tachyc. } \\
T_{I I, I I, a V F, V 4-6} l\end{array}$ & + \\
\hline 6 & $\mathrm{~T} . \mathrm{K}$. & 71 & $F$ & Cancer of Pancreas & $Q_{I I, M I}, S T_{I I, I I, a V F, V s, 6} \uparrow$ & H \\
\hline 7 & T. H. & 31 & M & Cancer of Stomach & Sinus Tachyc. & \\
\hline 8 & Y. H. & 66 & M & Hepatitis, Pneumonia & $\begin{array}{l}\mathrm{ST}_{\mathrm{V}_{4-6} \uparrow} \uparrow \text {, Giant Negative } T \text {, Sinus } \\
\text { Tachyc. }\end{array}$ & + \\
\hline 9 & K. K. & 67 & M & Cancer of Stomach & RBBB, Sinus Tachyc., Low Voltage & \\
\hline 10 & A. S. & 47 & M & Complete AV Block & Pacemaker Rhythm & \\
\hline 11 & M. M. & 71 & $\mathbf{F}$ & Cancer of Gall Bladder & Sinus Tachyc., Low Voltage & \\
\hline 12 & F. O & 73 & $\mathbf{M}$ & Cancer of Stomach, Sepsis & Sinus Tachyc., Low Voltage & \\
\hline 13 & A. $\mathrm{M}$. & 79 & $\mathbf{F}$ & Cancer of Colon & Trans. $\mathrm{ST}_{\mathrm{Y} 3-6} \hat{\jmath}, \mathrm{ST}, \mathrm{T}_{\mathrm{V} 3-6} \downarrow \downarrow$ & + \\
\hline
\end{tabular}

Abbreviations : LBBB ; complete left bundle branch block, af; atrial fibrillation, VT ; ventricular tachycardia, RBBB; complete right bundle branch block, D.M.; Diabetes mellitus.

Remark: \#; ECG changes were consistent with autopsy findings. +; ECG changes were not consistent with autopsy findings, or infarction-like ECG changes were transient. 
coronary artery and right coronary artery, resulting in a massive large lateral infarction (Case 2). In the 3rd case, there were large antero-septo-inferior infarctions with thrombi in the peripheral epicardial branches and multiple microthrombi in the intramyocardial arteries.

Clinical diagnosis of myocardial infarction was possible in 3 cases by electrocardiograms (Table III). Transient infarction-like electrocardiographic patterns were noted in 3 cases. Sinus tachycardia was the only change in 1 case of small myocardial infarction (Case 7). In 6 cases of small necrosis, transient ST elevation was observed in 2, and sinus tachycardia and low voltage in 3 . No specific diagnosis was made in 1 case with an implanted pacemaker.

IV. Coagulation tests and fresh myocardial lesions (Table IV)

Four kinds of coagulation tests were compared in 3 groups with or without necrosis and thrombosis. Platelet count, fibrinogen, and ELT did not show any significant correlations. But FDP was significantly higher in the group of coronary thrombosis than those without thrombosis, irrespective of presence or absence of myocardial necrosis.

\section{Representative cases (Tables II and III)}

Case 1 (T.S.) was a 74-year-old woman, who entered the hospital because of chest discomfort, cyanosis, and hypertension, in September, 1973. About 10 years ago hypertension was noted and had been treated. Five years prior to her admission she had an attack of cerebrovascular accident with left hemiparesis. Electrocardiograms on admission showed normal sinus rhythm, left ventricular hypertrophy, coronary $\mathrm{T}$ waves in the left precordial leads, and small QS in lead $\mathrm{aV}_{\mathrm{L}}$ (Fig. 1). Chest $\mathrm{X}$-ray films revealed marked bronchopneumonia. On October 18th, 1973, the electrocardiogram showed QS pattern in lead $V_{4}$. Platelet count decreased from $31 \times 10^{4} / \mathrm{mm}^{3}$ to $16 \times 10^{4} / \mathrm{mm}^{3}$, fibrinogen from 240 to $110 \mathrm{mg} / 100 \mathrm{ml}$, and FDP increased to $20 \mu \mathrm{g} / \mathrm{ml}$ and she died on October 22nd with an excretion of tarry stool.

Table IV. Coagulation Tests and Coronary Thrombosis in DIC

\begin{tabular}{|c|c|c|c|c|c|c|}
\hline & $\begin{array}{l}\text { Necrosis } \\
\text { Thrombosis }\end{array}$ & $\begin{array}{l}(+) \\
(+)\end{array}$ & $\begin{array}{l}\text { Necrosis } \\
\text { Thrombosis }\end{array}$ & $\begin{array}{l}(+) \\
(-)\end{array}$ & $\begin{array}{l}\text { Necrosis } \\
\text { Thrombosis }\end{array}$ & $\begin{array}{l}(-) \\
(-)\end{array}$ \\
\hline Platelet $\quad\left(\times 10^{4}\right)$ & $6.0 \pm 4.7$ & (13) & $9.3 \pm 15.0$ & (13) & $7.0 \pm 8.6$ & $(50)$ \\
\hline Fibrinogen $(\mathrm{mg} / 100 \mathrm{ml})$ & $138 \pm 90$ & (13) & $156 \pm 95$ & (12) & $143 \pm 81$ & (34) \\
\hline ELT $\quad$ (hrs) & $43 \pm 49$ & $(12)$ & $26 \pm 28$ & (11) & $24 \pm 22$ & $(31)$ \\
\hline$(\mu \mathrm{g} / \mathrm{ml})$ & $62 \pm 53^{*}$ & $(16)$ & $28 \pm 20^{* *}$ & $(10)$ & $22 \pm 20^{* * * *}$ & (28) \\
\hline
\end{tabular}




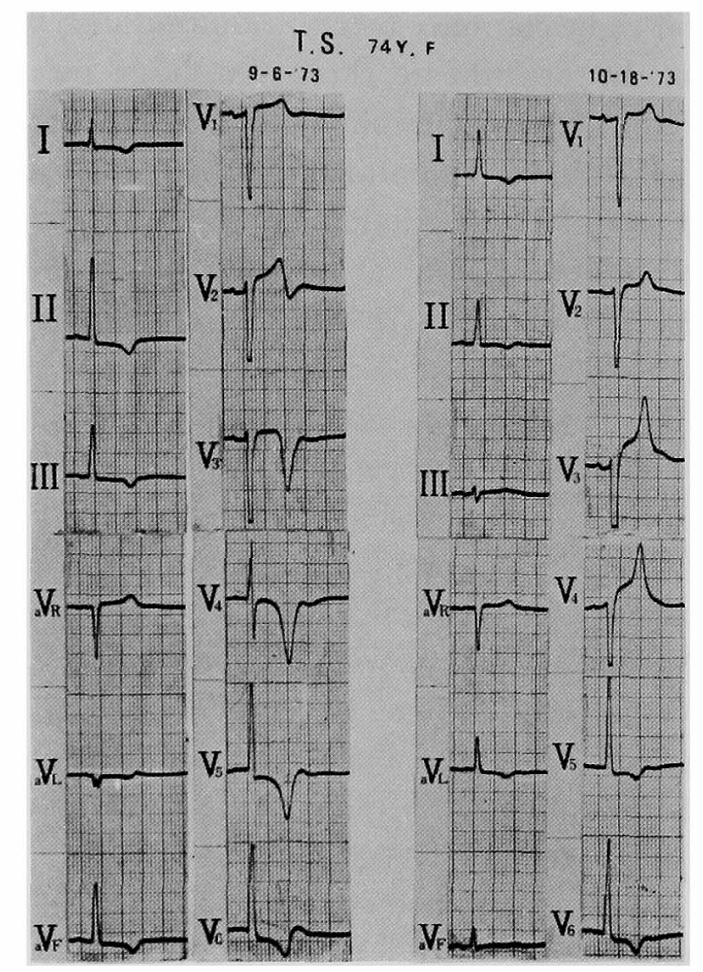

Fig. 1. Electrocardiograms of Case 1.

Heart weight was $420 \mathrm{Gm}$, with coronary stenotic index of 13 . All 3 vessels showed more than $75 \%$ stenosis, and the left anterior descending artery was completely occluded by a fresh thrombus of $0.8 \mathrm{~mm}$ in diameter, resulting in a large fresh anterior myocardial infarction (Fig. 2).

Case 2 (K.T.) was an 85-year-old man, who was admitted to the hospital with a chief complaint of melena in January, 1973. He was diagnosed to have a cancer of stomach in September, 1972, but no surgical treatment was made due to reluctance of his family. Electrocardiograms on admission showed normal sinus rhythm and QS in leads $\mathrm{V}_{1}$ to $\mathrm{V}_{4}$, suspecting old anteroseptal infarction. Platelet count decreased from $19 \times 10^{4}$ to $7 \times 10^{4}$ and $0.8 \times 10^{4} / \mathrm{mm}^{3}$, fibrinogen from 118 to less than $100 \mathrm{mg} / 100 \mathrm{ml}$, and FDP was 160,80 , and $10 \mu \mathrm{g} / \mathrm{ml}$ in the end of February, March, and May, respectively. He died of bronchopneumonia on May 4th, 1973. Electrocardiograms were the same throughout the clinical course. Heart weighed $460 \mathrm{Gm}$. All of 3 vessels were devoid of any marked atherosclerosis, but peripheral regions of the left circumflex coronary artery and right coronary artery showed thrombosis and also intramyocardial branches were occluded by thrombi (Fig. 3 ), resulting in a large lateral infarction. Histological examination showed 


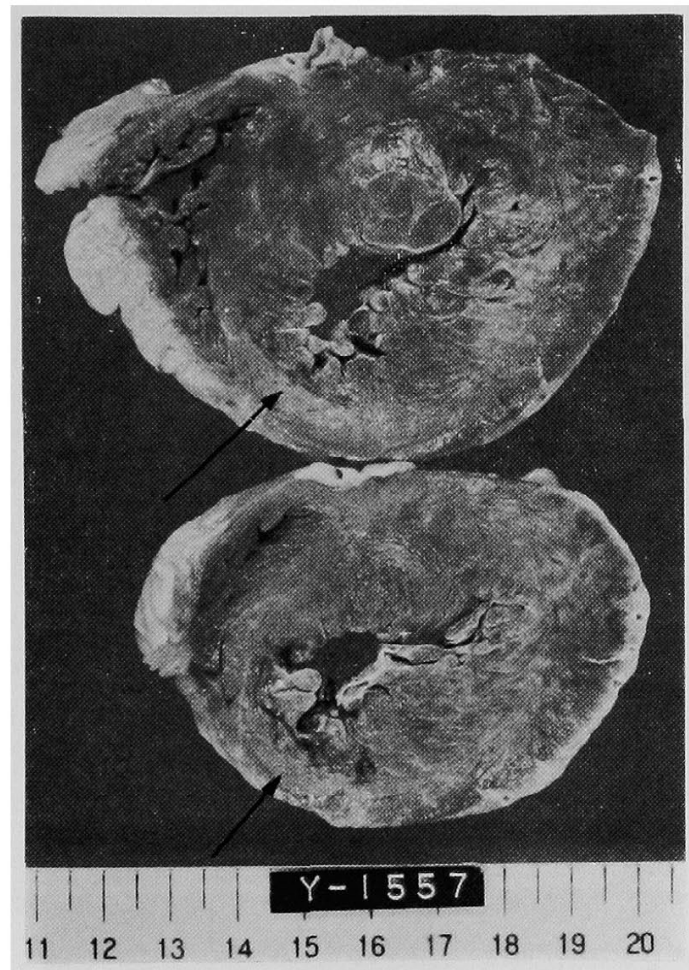

Fig. 2. Anterior infarction found in Case 1, which was mainly subendocardial, and partly involving more than half of the wall thickness (arrows).

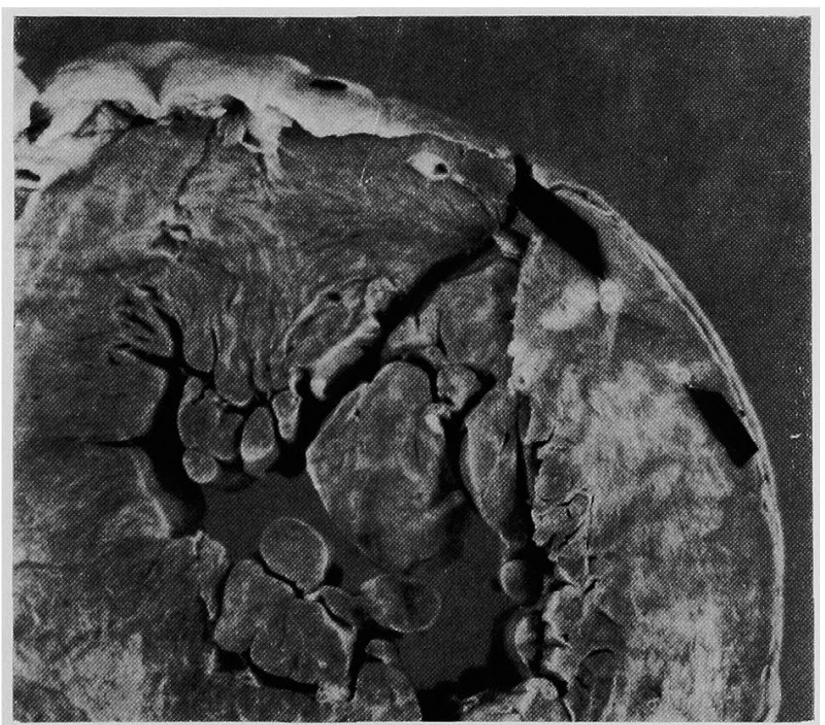

Fig. 3. Latero-inferior infarction found in Case 2, with multiple occluded small arteries (arrows). 
multiple microthrombi in the intramyocardial arteries (Fig. 4). Myocardial lesions were not uniform, consisting of old and new ischemic foci, suggesting a prolonged course of myocardial ischemia.

Case 6 (T.K.) was a 71-year-old woman, who entered the hospital because of sudden onset of left hemiplegia and coma. ECG on admission was within normal limits, platelet count was $9.5 \times 10^{4} / \mathrm{mm}^{3}$, fibrinogen $250 \mathrm{mg}$ / $100 \mathrm{ml}$, and FDP $20 \mu \mathrm{g} / \mathrm{ml}$. On December 31st, 1973, she complained of chest pain. ECG on January 1st, 1974 showed $Q$ waves and ST elevation in leads II, III, and $\mathrm{aV}_{\mathrm{F}}$ with a diagnosis of inferior infarction (Fig. 5). On January 4th, 1974, platelet count was $4.7 \times 10^{4} / \mathrm{mm}^{3}$, fibrinogen $100 \mathrm{mg} / 100 \mathrm{ml}$, and FDP was $20 \mu \mathrm{g} / \mathrm{ml}$, and she died on January 9th due to shock. Autopsy disclosed a cancer of the pancreas. Heart weight was $230 \mathrm{Gm}$ and coronary sclerosis was slight. The periphery of the left circumflex coronary artery had a thrombus of $2.1 \mathrm{~mm}$ in diameter (Fig. 6) and also there were multiple microthrombi in the intramyocardial coronary arteries, resulting in a middlesized fresh myocardial infarction at the inferior wall (Fig. 7).

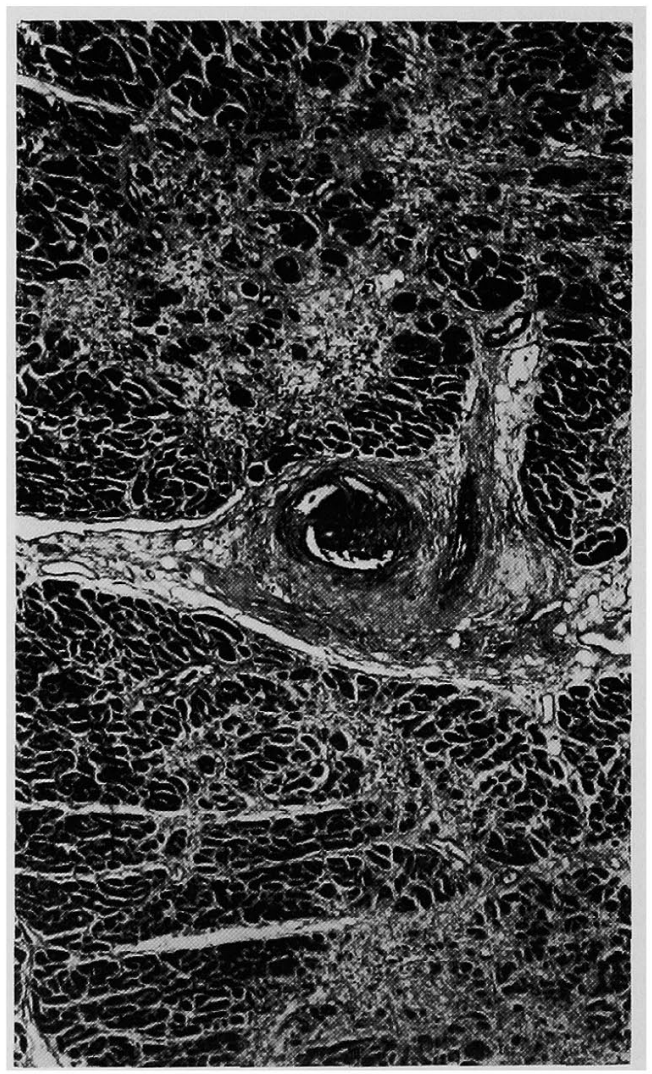

Fig. 4. Thrombotic occlusion of the intramyocardial coronary arteries and myocardial fibrosis in Case $2(\mathrm{H} . \mathrm{E} . \times 40)$. 


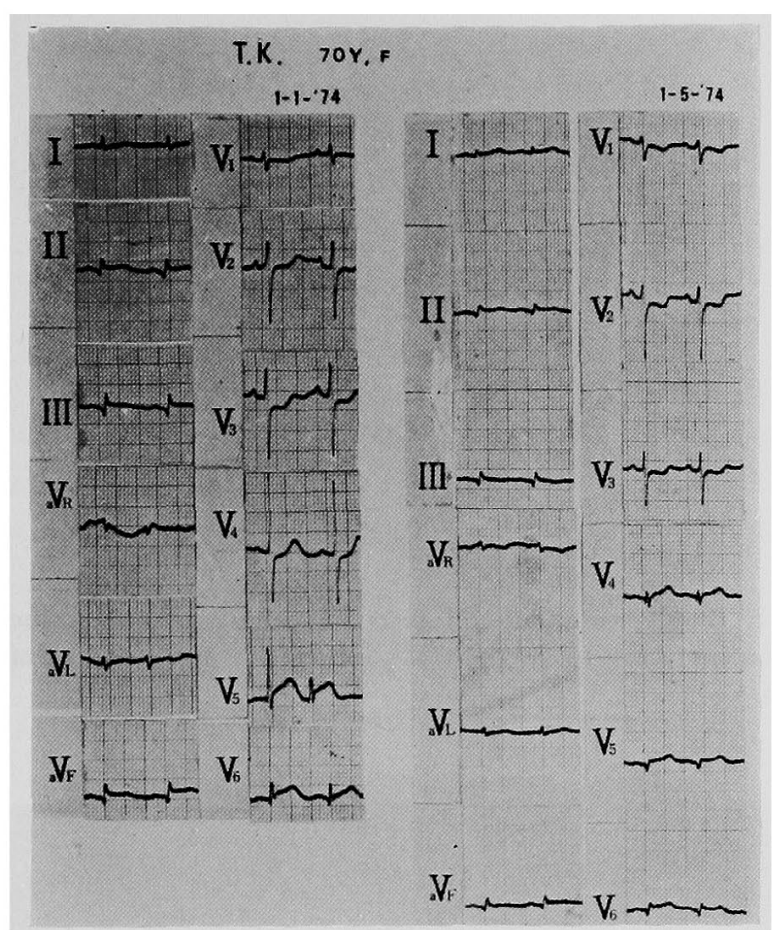

Fig. 5. Electrocardiograms of Case 6 .

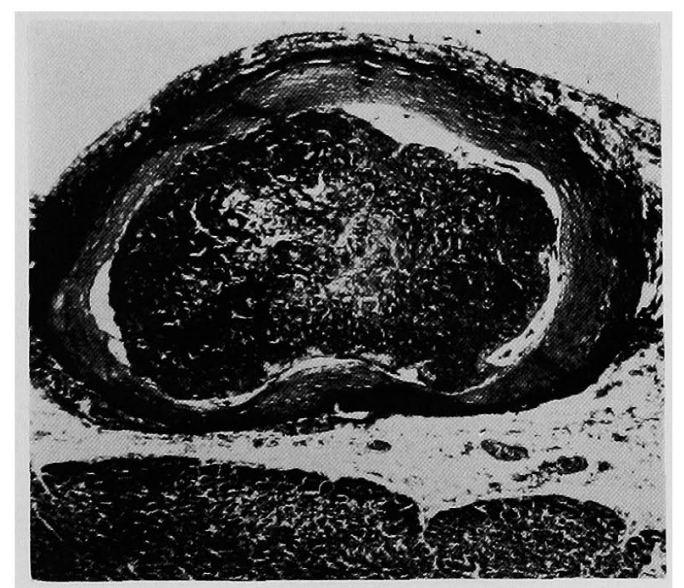

Fig. 6. Thrombotic occlusion of the peripheral branch of the left circumflex coronary artery with only slight atherosclerosis in Case 6 (Azan $\times 20$ ).

Fig. 8 was obtained from Case 9, which showed multiple small necrosis mainly at the inferior wall. Fig. 9 was a transverse section of the heart in Case 12, showing vortexlike hemorrhage in the myocardium. Fig. 10 showed nonbacterial thrombotic endocarditis at the mitral valve in Case 5. 


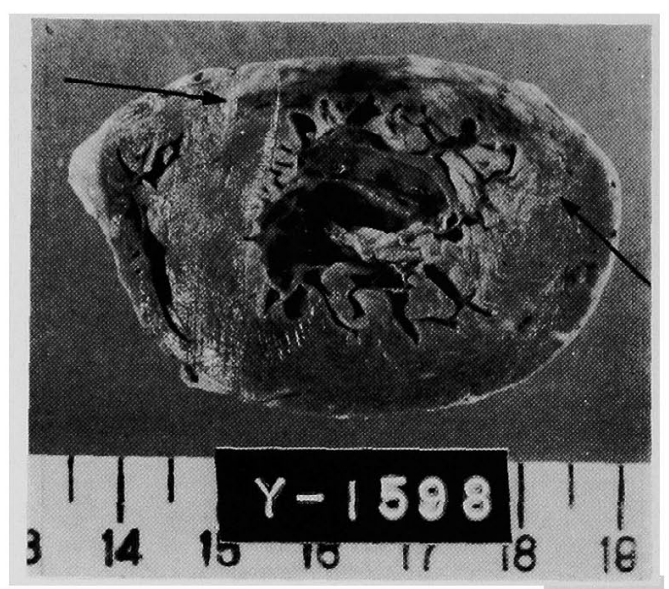

Fig. 7. Fresh myocardial infarction (between 2 arrows) at the inferior wall of the left ventricle, with myocardial bleeding and thinning of the wall in Case 6.

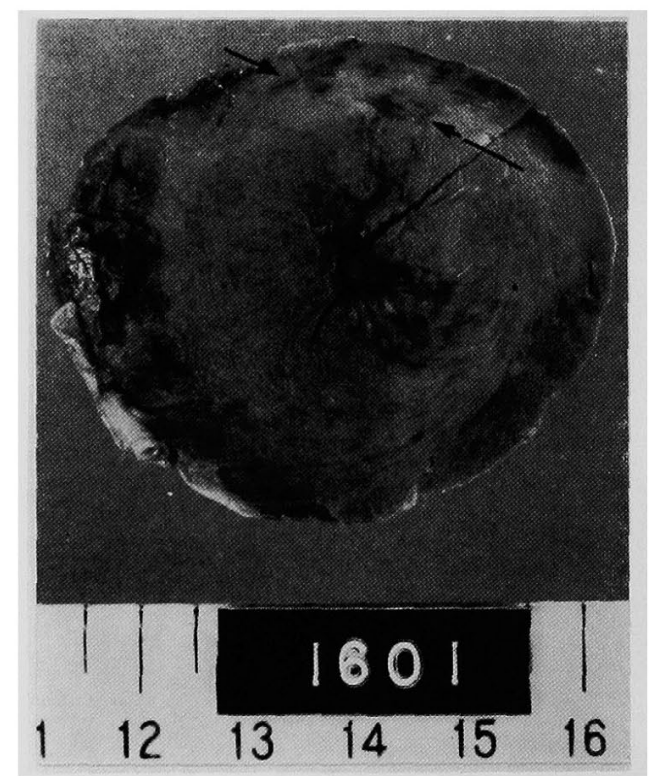

Fig. 8. Scattered small necrosis in Case 9 (arrows).

\section{Discussion}

There have been an accumulation of reports on DIC, but its clinicopathological correlation studies were not enough especially in the cardiac lesions. ${ }^{1,5), 6)}$ Robboy et ${ }^{(5)}$ found cardiac lesions in 6 cases $(27.3 \%$ ) out of 22 autopsy cases of DIC, consisting of 2 mural thrombi, 2 marantic endocarditis and 3 coronary thrombosis, and there was no myocardial infarction. 


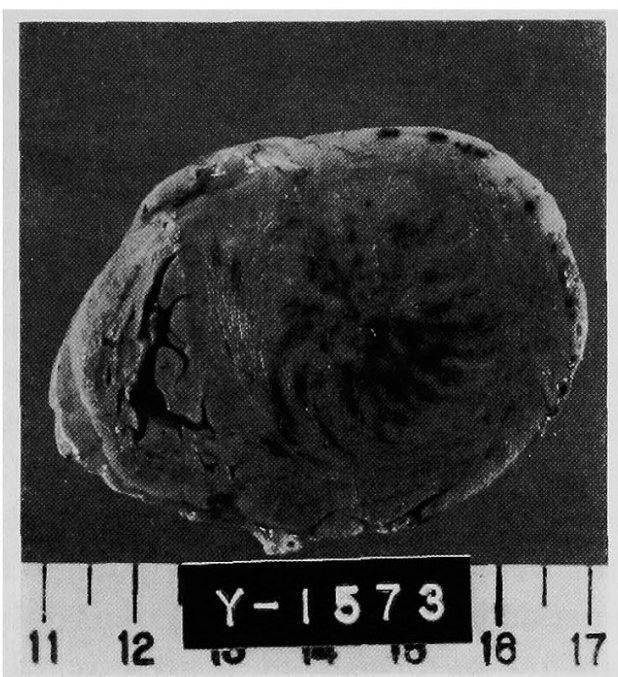

Fig. 9. Myocardial bleeding found in Case 12 .

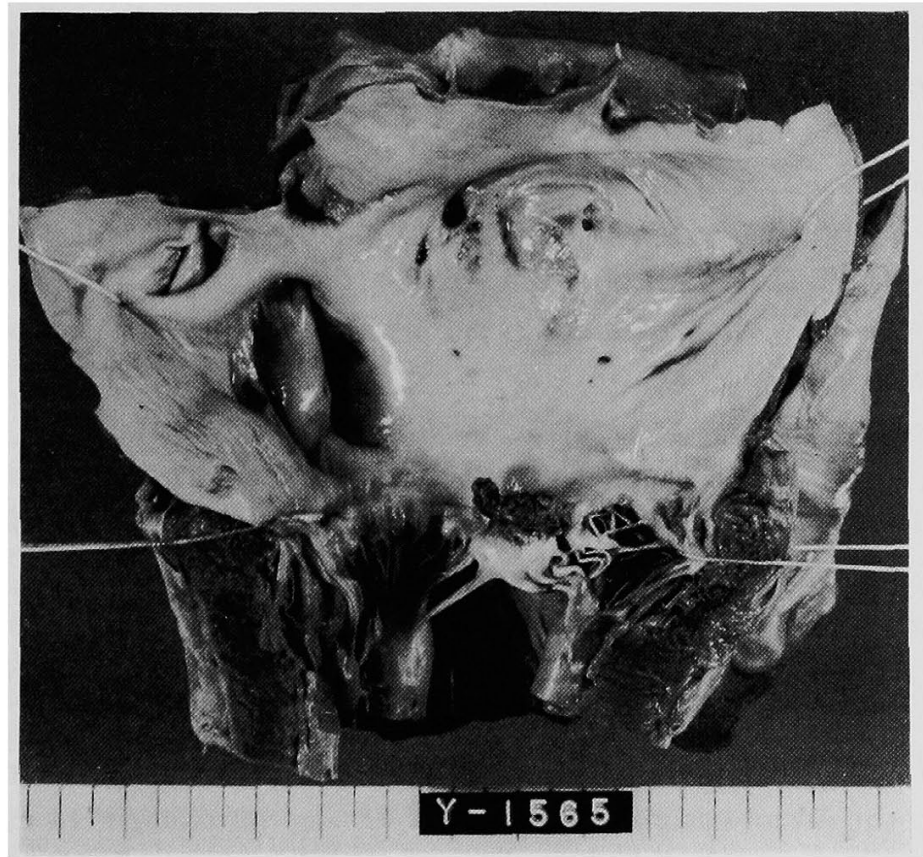

Fig. 10. Nonbacterial thrombotic endocarditis found in Case 5 (anterior leaflet of the mitral valve).

This study revealed that fresh lesions of the heart were unexpectedly frequent (62.5\% among total DIC), fresh myocardial necrosis in $37.5 \%$ and especially acute myocardial infarction in $14.1 \%$. The incidence of myocardial infarction in consecutive autopsy was $13.7 \%$ among 1,000 aged cases, and fresh in- 
farction was only $3.5 \%{ }^{8}$ ) Comparison of these 2 series on the incidence of myocardial infarction disclosed that DIC had a higher incidence. Polliack ${ }^{6}$ reported 3 cases of DIC with acute myelocytic leukemia; 2 of them showed bleeding in the various portions of the heart, but no thrombosis. In our study the incidence of cardiac bleeding was $17.2 \%$.

Clinical significance was high in occurrence of myocardial infarction among cardiac lesions of DIC. In severe coronary atherosclerosis, thrombus of small size could cause large infarction, which could not be distinguished from usual myocardial infarction, except the background of accelerated intravascular coagulation in its pathogenesis (Cases 1 and 4 in Tables II and III). Basic atherosclerosis was not necessarily marked in DIC. Thrombi in the peripheral arteries or the branches were generally small up to $2 \mathrm{~mm}$ in diameter, but the scale of thrombus formation in these arteries determined the size of myocardial necrosis, from large infarction to small focal necrosis. This type of necrosis was found in 5 cases of myocardial infarction (Cases 2,3,5,6 and 7 in Table II). Though the clinical significance was less important, thrombus formation at the level of intramyocardial small arteries or arterioles induced scattered necrosis without any rule in its distribution (Cases 8 to 13 in Table II). A case of DIC with sudden death was examined to show an extensive thrombus formation and infarction of the sinoatrial node. ${ }^{9)}$

Above-mentioned 13 cases had thrombosis in the various levels of coronary arteries and corresponding myocardial necrosis. From the standpoint of coagulation study, FDP was significantly higher in this group than in the group without coronary thrombosis.

A group of myocardial necrosis without coronary thrombosis was found in 11 cases, consisting of 2 cases of myocardial infarction and 9 cases of small necrosis. There were no differences in myocardial lesions, except for absence of coronary thrombosis. Absence of coronary thrombosis could be due to (1) limit in the number of preparations (possibility of presence of coronary thrombosis in somewhere besides observed preparations), or (2) true absence of coronary thrombosis (absence of thrombi throughout the course or thrombolysis). These cases were very similar to the reported cases of myocardial infarction in the intact coronary arteries. ${ }^{10)-12}$ In our study ${ }^{10 /} 14$ cases among 188 cases of myocardial infarction showed almost intact coronary arteries $(7.45 \%)$. The range and location of these infarctions strongly suggested the presence of thrombi in the past. This group needs further study in the future.

Nonbacteral thrombotic endocarditis has been known to occur in the marantic diseases including malignancy and severe infections. Rosen and Armstrong ${ }^{13)}$ reported 75 cases of endocarditis in malignancy, and 4 of them showed marked decrease of platelet count. But their description on the coag- 
ulation study was not detailed, and relation to the DIC was not clear. In a case of DIC, marantic endocarditis was assumed to be the origin of multiple embolic phenomena. ${ }^{14)}$ Waller et al $^{15}$ reported systemic embolism in $34 \%$ of 32 cases of marantic valvular vegetations, but they did not refer to DIC nor coagulation study. In our study the incidence of valvular vegetations was $26.6 \%$, and in 8 cases out of 17 vegetations $(47 \%$ ) coronary thrombosis was found, where differentiation of embolism and thrombosis was hardly possible, especially when its distribution was diffuse. This is also the new theme, which needs further re-evaluation from the standpoint of DIC.

This paper was presented at the 39th Annual Meeting of Japanese Circulation Society, on April 2, 1975.

\section{REFERENCES}

1. McKay DG: Progress in disseminated intravascular coagulation. California Med 111 : 186 , 1969

2. Colman RW, Robboy SJ, Minna JD: Disseminated intravascular coagulation. An approach. Am J Med 52: 679, 1972

3. Minna JD, Robboy SJ, Colman RW: Disseminated intravascular coagulation in man. Charles G Thomas Publ, Springfield, 1974

4. Matsuda T: Intravascular coagulation. Nihon-Rinsho 32: 979, 1974 (in Japanese)

5. Robboy SJ, Colman RW, Minna JD: Pathology of disseminated intravascular coagulation. Analysis of 26 cases. Human Pathol 3: 327, 1972

6. Polliack A: Acute promyelocytic leukemia with disseminated intravascular coagulation. Am J Clin Path 56: 155, 1971

7. Sugiura M, Okada R: A clinicopathological study on the natural history of myocardial infarction in the aged. Jap Circulat J 36: 2, 1972

8. Sugiura M, Hiraoka K, Ohkawa S, Shimada H: A clinicopathological study on the heart diseases in the aged. The morphological classification of the 1,000 consecutive autopsy cases. Jap Heart. J 16: 526, 1975

9. James TN, Marshall ML, Craig MW: De Subitaneis Mortibus: VII Disseminated intravascular coagulation and paroxysmal atrial tachycardia. Circulation 50: 395, 1974

10. Sugiura $\mathbf{M}$, Hiraoka $\mathrm{K}$, Ohkawa S: Myocardial infarction from intact coronary arteries. Sogo-Rinsho $23: 340,1974$ (in Japanese)

11. Friedberg CK, Horn $\mathrm{H}$ : Acute myocardial infarction not due to coronary artery occlusion. JAMA 112: 1675, 1939

12. Gross H, Sternberg WH: Myocardial infarction without significant lesions of coronary arteries. Arch Int Med 64: 249, 1939

13. Rosen $\mathrm{P}$, Armstrong D: Nonbacterial thrombotic endocarditis in patients with malignant neoplastic diseases. Am J Med 54: 23, 1973

14. Washington University School of Medicine: Marantic endocarditis (GPC). Am J Med 56: 871,1974

15. Waller BF, Knapp WS, Edwards JE: Marantic valvular vegetations (CPC). Circulation 48: 644,1973 